\title{
Strong-coupling diagnostics for multimode open systems
}

\author{
C. Kow, ${ }^{1, *}$ Z. Xiao, ${ }^{1, *}$ A. Metelmann, ${ }^{2, \dagger}$ and A. Kamal ${ }^{1}$ \\ ${ }^{1}$ Department of Physics and Applied Physics, University of Massachusetts, Lowell, Massachusetts 01854, USA \\ ${ }^{2}$ Department of Electrical Engineering, Princeton University, Princeton, New Jersey 08544, USA
}

(Received 1 October 2020; accepted 30 March 2021; published 3 May 2021)

\begin{abstract}
Engineered quantum systems play a central role in quantum information processing. Typically, these systems are strongly coupled to their environments, which presents challenges and opportunities to harness controllable quantum effects in such systems. One of the outstanding challenges is to characterize the quantum correlations that develop in such out-of-equilibrium quantum systems. Here we present a method that utilizes low-dimensional projections of eigenvectors to distinguish different types of correlations in an $N$-mode open system. The proposed method presents a nontrivial extension of eigenvalue-based analysis employed for such systems; specifically, it not only diagnoses crossovers between weak and strong coupling regimes (also known as "exceptional points") but also can identify the physical systems of interest spanning the correlated subspaces in different regions of the multimode strong-coupling regime. As a demonstration of this feature, we apply our method to study hybridization physics in a three-mode optomechanical system and determine the parameter regime for efficient sideband cooling of the system in the presence of reservoir correlations.
\end{abstract}

DOI: 10.1103/PhysRevResearch.3.023093

\section{INTRODUCTION}

Strongly coupled open systems form the operational framework in diverse fields, ranging from quantum information processing and precision measurements to quantum chemistry. One of the main challenges in modeling such systems is the appearance of strongly hybridized dressed states beyond a critical coupling strength, which necessitates describing dissipative dynamics in a nonlocal basis. A powerful framework for analyzing this transition from weak to strong coupling in open systems is provided by exceptional points (or EPs). EPs are branch point singularities in the parameter space, where two (or more) eigenvalues and eigenstates of the system coalesce. This makes them distinct from degeneracy points in Hamiltonian systems, which support identical eigenvalues while corresponding eigenvectors remain orthogonal. The physics of EPs continues to be exploited in a variety of applications involving non-Hermitian physics, such as nonreciprocal devices [1-3], amplifiers [4,5], quantum sensors [6-8], and single-mode lasers [9-11], to name a few.

Though EPs represent points where both eigenvalues and eigenvectors collapse to a single value, the analysis and design of open systems utilizing EPs predominantly makes use of eigenvalues of the dynamical matrix [12]. This is rooted in

\footnotetext{
*These authors contributed equally to this work.

†resent address: Dahlem Center for Complex Quantum Systems and Fachbereich Physik, Freie Universität Berlin, 14195 Berlin, Germany.

Published by the American Physical Society under the terms of the Creative Commons Attribution 4.0 International license. Further distribution of this work must maintain attribution to the author(s) and the published article's title, journal citation, and DOI.
}

the fact that the nontrivial topological properties associated with the emergence of such degeneracies, such as nonadiabatic mode switching [13] and chiral state transfer [14], can be entirely described by tracking the eigenvalues alone in the complex parameter space [15]. In this paper, our focus is quite different: Rather than study the properties of the dressed states, we aim to study the strong-coupling physics from the point of view of physical subsystems. To this end, we present a method that shows how eigenvectors can provide a comprehensive description of strong-coupling effects in open systems. The basic idea relies on exploiting the mode correlations as reflected by the eigenvector projections in relevant subspaces of an $\mathrm{N}$-dimensional mode space. Our proposed method goes beyond the usual identification of EPs that delineate weak- and strong-coupling regimes and provides more nuanced information about different types of correlations in a multimode open system under strong coupling. Most importantly, it provides a means to identify the physical modes that hybridize to form the dressed eigenstates (also referred to as "supermodes"), a feature not accessible with eigenvalue-based analysis or EPs alone. We emphasize the physical significance of such subsystem identification in strong-coupling manifolds, using the example of cooling of a mechanical oscillator to its quantum ground state using engineered dissipation. The proposed criterion enables characterization of the operational cooling regime, where the system oscillator remains weakly coupled to strongly correlated reservoir modes.

The paper is organized as follows: We begin with a description of an $N$-mode open system with nearest neighbor interactions in Sec. III and use $N=3$ and $N=4$ cases as examples to illustrate the inadequacy of conventional eigenvalue-based analysis when extended to more than two modes. We then introduce the eigenvector projection-based method in Sec. III and show how it can be used to generate the 


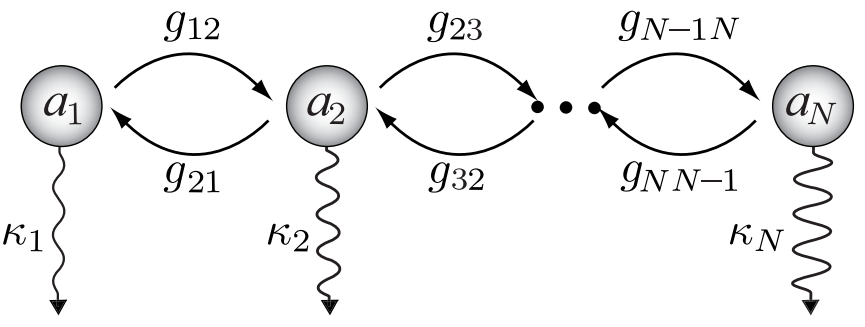

FIG. 1. Schematic of an $N$-mode open system with nearest neighbor interactions. Curved arrows depict the bilinear interactions $g_{i j}$, while local decay rates of the modes are depicted with $\kappa_{j}$.

detailed coupling map of a multimode open system, resolving the shortcomings of the usual method restricted to identification of EPs. In Sec. IV, we examine quantum ground-state cooling in a three-mode optomechanical system to show how the proposed method can be applied to a physical problem of interest. We conclude with a summary of main results and offer perspectives for potential extensions of our study in Sec. V. Additional calculation details are included in Appendices A and $\mathrm{B}$.

\section{EXCEPTIONAL POINTS IN A MULTI-MODE SYSTEM}

A generic $N$-mode open system with nearest neighbor hopping interactions, as depicted in Fig. 1, can be described by a Hamiltonian of the form

$$
\mathbb{H}^{(N)}=\sum_{\substack{j=1, k=1 \\\langle j, k\rangle}}^{N}\left(\frac{\Delta_{j}}{2} \delta_{j, k}+g_{j k}\left(1-\delta_{j, k}\right)\right) a_{j}^{\dagger} a_{k},
$$

written in the interaction frame defined with respect to the Hamiltonian $\sum_{j} \omega_{j}^{d} a_{j}^{\dagger} a_{j}$, with $\Delta_{j}=\omega_{j}-\omega_{j}^{d}$ being the detunings associated with each mode. The phase of the couplings is determined by $\arg \left(g_{j k}\right)$, with $g_{j k}=g_{k j}^{*}$ ensuring Hermiticity of the interaction Hamiltonian. The open dynamics of this system can be derived from Heisenberg-Langevin equations for the mode annihilation operators, $a_{j}$, as

$$
\frac{d \mathbf{V}^{(\mathrm{N})}}{d t}=\mathbb{M}^{(\mathrm{N})} \mathbf{V}^{(\mathrm{N})}+\sqrt{\mathbb{K}^{(\mathrm{N})}} \mathbf{V}^{\mathrm{in}(\mathrm{N})},
$$

where $\mathbf{V}^{(\mathrm{N})}=\left[a_{1}, a_{2}, \ldots, a_{N}\right]^{\mathrm{T}}, \mathbf{V}^{\text {in(N)}}=\left[a_{1}^{\text {in }}, a_{2}^{\text {in }}, \ldots, a_{N}^{\text {in }}\right]^{\mathrm{T}}$ denote the internal mode and input noise operators respectively, and $\mathbb{K}^{(\mathrm{N})}=\operatorname{diag}\left(\kappa_{1}, \ldots, \kappa_{j}, \ldots, \kappa_{N}\right)$ is a diagonal matrix with its nonzero elements representing the decay rates associated with each individual modes. The dynamical matrix $\mathbb{M}^{(\mathrm{N})}$, also referred to as the "mode matrix", for the system with nearest neighbor couplings considered here is an $N \times N$ tridiagonal complex-symmetric matrix of the form

$$
\mathbb{M}^{(\mathrm{N})}=\left(\begin{array}{ccccc}
\widetilde{\Delta}_{1} & -i g_{12} & 0 & \ldots & 0 \\
-i g_{21} & \widetilde{\Delta}_{2} & -i g_{23} & \ldots & 0 \\
0 & -i g_{32} & \widetilde{\Delta}_{3} & \ldots & 0 \\
\vdots & \vdots & \vdots & \ddots & \vdots
\end{array}\right),
$$

where $\widetilde{\Delta}_{j} \equiv-i \Delta_{j}-\kappa_{j} / 2$. Note that here we have assumed open boundary conditions; closed-loop topologies with periodic boundary conditions have been studied in the past and while they can support qualitatively new physics, the shape of coupling map is not germane to the question of diagnosing strong coupling that we focus on in the following sections.

Conventionally, weak and strong coupling regimes are identified by finding the exceptional points (EPs) supported by $\mathbb{M}^{(N)}$. For instance, for the well-known case of two modes coupled with a hopping-type interaction, an EP2 is realized for $g_{\mathrm{EP} 2}^{(2)}=\left|\kappa_{1}-\kappa_{2}\right| / 4$. In the weak coupling regime, with $g<g_{\mathrm{EP} 2}^{(2)}$, the eigenvalues are purely real, while in the strong-coupling regime, with $g>g_{\mathrm{EP} 2}^{(2)}$, the eigenvalues become complex; the imaginary part corresponds to the detuning of the mode from resonance due to hybridization that lifts the degeneracy, manifesting as a "splitting" of the mode spectrum. In general, this transition between real and complex solutions (or EP2) for an $N$-mode system can be obtained by setting the discriminant of the characteristic polynomial of the mode matrix, $p_{\mathbb{M}^{(N)}}=\operatorname{det}\left(\lambda \mathbb{I}-\mathbb{M}^{(N)}\right)$, to zero,

$$
\operatorname{disc}\left(p_{\mathbb{M}^{(N)}}\right) \equiv \Pi_{\alpha \neq \beta}\left(\lambda_{\alpha}-\lambda_{\beta}\right)=0,
$$

where $\lambda_{\alpha, \beta}$ denote a pair of eigenvalues [16]. Since $p_{\mathbb{M}^{(N)}}$ is a polynomial of degree $N$ in $\lambda$, the strong coupling regime needs to be studied in a hyperplane spanned by $N-1$ coupling parameters $g_{i j}$ for fixed values of decay rates $\kappa_{j}$. As concrete examples, we now consider $N=3$ and $N=4$ systems depicted in Fig. 2(a) in detail and describe the generic features of EPs in systems with bilinear interactions.

$$
\text { A. } N=3 \text { case }
$$

Time-averaged dynamics of a three-mode open system with nearest neighbor couplings can be described by a $3 \times 3$ mode matrix of the form

$$
\mathbb{M}^{(3)}=\left(\begin{array}{ccc}
-\kappa_{1} / 2 & -i g_{1}^{(3)} & 0 \\
-i g_{1}^{(3)} & -\kappa_{2} / 2 & -i g_{2}^{(3)} \\
0 & -i g_{2}^{(3)} & -\kappa_{3} / 2
\end{array}\right)
$$

where $g_{12}=g_{1}^{(3)}, g_{23}=g_{2}^{(3)}$, with $g_{j}^{(3)} \in \mathbb{R}_{>0} \forall j$. Here, without loss of generality, we have considered resonant driving, leading to zero detunings, i.e., $\Delta_{j}=0, \forall j$. Figure 2 (b) shows a plot of EP2s for this system as a function of the interaction strengths, obtained from Eq. (4) for fixed values of decay rates $\kappa_{j}$. Analogous to the two-mode setup, we note that the EP2s demarcate the weak- and strong-coupling regimes; specifically, the intercepts on the $x$ and the $y$ axes correspond to $\gamma_{-}=\left|\kappa_{1}-\kappa_{2}\right| / 4$ and $\kappa_{-}=\left|\kappa_{2}-\kappa_{3}\right| / 4$ respectively, which are the EP2 thresholds for decoupled $\left\{a_{1}, a_{2}\right\}$ and $\left\{a_{2}, a_{3}\right\}$ subsystems in the absence of other couplings. We emphasize that within the region bounded by the EP2 curves, the system is in the weak-coupling regime with purely real eigenvalues, whereas outside this region all three eigenvalues can be complex and the system is in the strong-coupling regime.

A noteworthy feature for systems with $N>2$ is the appearance of higher order exceptional points. For instance, as shown in Fig. 2(b), a three-way exceptional point (EP3) is realized at the coincidence of two EP2 curves, where all three eigenvalues and eigenvectors become identical. The coordinates of EP3 in the coupling phase diagram are 
(a)

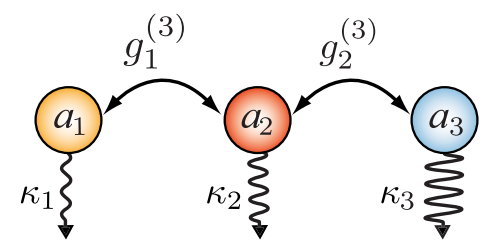

(b)

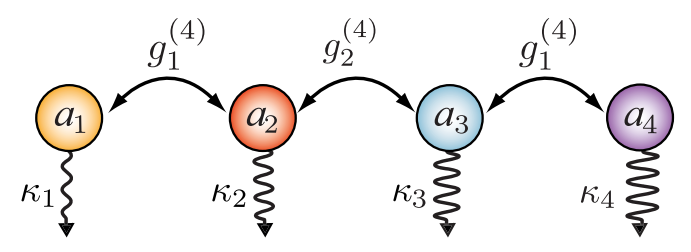

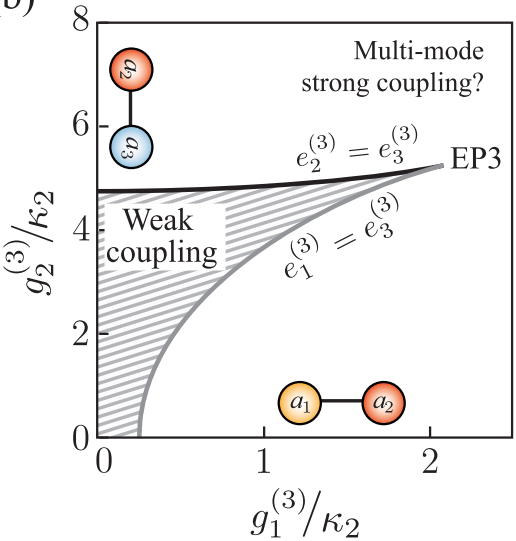

(c)

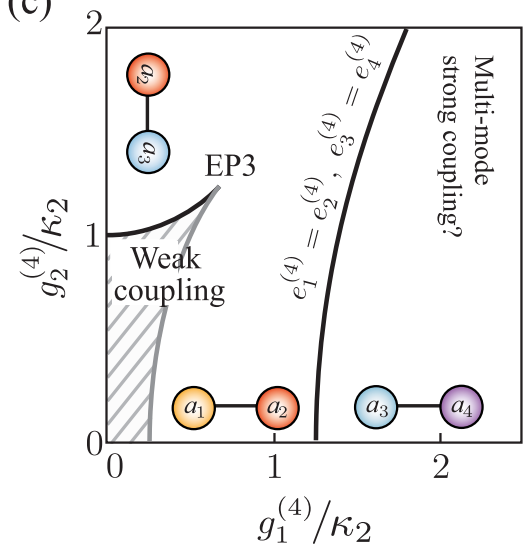

FIG. 2. (a) Illustration of three-mode $(N=3)$ and four-mode $(N=4)$ systems with nearest-neighbor hopping interactions. The hopping interactions are depicted with double-headed arrows $g_{j}^{(N)}, j \in[1, N-1]$, and local decay rates are represented as wavy arrows $\kappa_{j}, j \in[1, N]$. [(b), (c)] Coupling phase diagrams for $N=3$ and $N=4$ systems obtained using eigenvalues of the respective mode matrix. The decay rates used in the calculation are $\kappa_{1} / \kappa_{2}=0.01$ and $\kappa_{3} / \kappa_{2}=20$ for $N=3$ case and $\kappa_{1} / \kappa_{2}=0.01, \kappa_{3} / \kappa_{2}=5$, and $\kappa_{4} / \kappa_{2}=10$ for $N=4$ case. In each plot, the solid curves denote the locus of EP2s in the parameter space, calculated using Eq. (4), and are labeled with the eigenvalues that coalesce at the respective EP. The hatched (white) region correspond to the weak-coupling (strong-coupling) regime. The insets near the axis pictorially depict the relevant pair of modes that hybridize when the coupling strength is increased beyond the corresponding EP2 threshold.

given by

$$
\begin{aligned}
& \left(\left.g_{1}^{(3)}\right|_{\mathrm{EP} 3},\left.g_{2}^{(3)}\right|_{\mathrm{EP} 3}\right) \\
& \quad=\left(\sqrt{\frac{4\left(2 \gamma_{-}+\kappa_{-}\right)^{3}}{27\left(\gamma_{-}+\kappa_{-}\right)}}, \sqrt{\frac{4\left(2 \kappa_{-}+\gamma_{-}\right)^{3}}{27\left(\gamma_{-}+\kappa_{-}\right)}}\right) .
\end{aligned}
$$

Note that the preceding analysis does not reveal exact nature of coupling between the modes or identify which modes are strongly coupled in the white region of Fig. 2(b); we can only ascertain that there exists at least one pair of modes that is strongly coupled for $\left(g_{1}^{(3)}>\gamma_{-}\right) \cap\left(g_{2}^{(3)}>\kappa_{-}\right)$. For explicit expressions for eigenvalues in a three-mode system, we refer the reader to Appendix A.

\section{B. $N=\mathbf{4}$ case}

We consider a four-mode system described by a mode matrix of the form

$$
\mathbb{M}^{(4)}=\left(\begin{array}{cccc}
-\kappa_{1} / 2 & -i g_{1}^{(4)} & 0 & 0 \\
-i g_{1}^{(4)} & -\kappa_{2} / 2 & -i g_{2}^{(4)} & 0 \\
0 & -i g_{2}^{(4)} & -\kappa_{3} / 2 & -i g_{1}^{(4)} \\
0 & 0 & -i g_{1}^{(4)} & -\kappa_{4} / 2
\end{array}\right) .
$$

As before, we consider resonant driving, with $g_{12}=g_{34}=$ $g_{1}^{(4)}, g_{23}=g_{2}^{(4)}$, where $g_{j}^{(4)} \in \mathbb{R}_{>0} \forall j$. Besides allowing us to restrict our analysis to a $2 \mathrm{D}$ phase diagram, this pattern of alternating couplings is of relevance to interesting physical models, such as the Su-Schrieffer-Heeger (SSH) model $[17,18]$ describing hopping of spinless fermions on a $1 \mathrm{D}$ lattice [19].

Proceeding as in the case of three modes, we obtain the EP2s of the four-mode system as a function of coherent couplings $g_{1,2}^{(4)}$ for fixed decay rates $\kappa_{j}$ (see Appendix A for details). As shown in Fig. 2(c), we now obtain three EP2 curves with three intercepts on the axes. The intercepts on the $x$ axis correspond to $g_{1}^{(4)}=\left|\kappa_{2}-\kappa_{1}\right| / 4$ and $g_{1}^{(4)}=\left|\kappa_{4}-\kappa_{3}\right| / 4$, setting the strong-coupling thresholds for decoupled $\left\{a_{1}, a_{2}\right\}$ and $\left\{a_{3}, a_{4}\right\}$ subsystems respectively. Similarly, the $y$ intercept corresponds to $g_{2}^{(4)}=\left|\kappa_{3}-\kappa_{2}\right| / 4$, the strong-coupling threshold for decoupled $\left\{a_{2}, a_{3}\right\}$ subsystem. However, as in the case for three modes, in regions sufficiently distant from the axes, the identity of the modes that are strongly coupled remains ambiguous.

\section{STRONG-COUPLING ANALYSIS BASED ON EIGENVECTORS}

As is evident from the discussion in the previous section, while EPs provide a clear separation of weak- and strongcoupling regimes, they fail to identify the physical modes that span the strongly coupled subspace in a multimode $(N>2)$ system past EPN [white region of Figs. 2(b) and 2(c)]. In this section, we introduce a method based on 2D planar projections of eigenvectors which provides a universal way to detect $N$-way hybridization, complete with an identification of the strongly coupled subspace, in a multimode open system.

We begin with a simple two-mode example to illustrate the behavior of eigenvectors in weak- and strong-coupling regimes. To this end, we consider the amplitudes of (normalized) left eigenvectors $V^{\alpha}$,

$$
\left|V^{\alpha}\right| \equiv\left[\left|\left(V^{\alpha}, a_{1}\right)\right|,\left|\left(V^{\alpha}, a_{2}\right)\right|\right],
$$

where $a_{j} \equiv\left[1_{j}\right]$ denotes a basis vector with unity as the $j$ th physical mode and zero for every other entry, and $(u, v)$ represents the vector inner product. The vector $\left|V^{\alpha}\right|$ can be thought of as a "participation ratio vector" since each entry denotes the participation ratio of physical mode $a_{i}$ in the eigenmode. For $g^{(2)}=0,\left|V^{1}\right|=a_{1}=[1,0]$ and $\left|V^{2}\right|=$ $a_{2}=[0,1]$; hence $\left(\left|V^{1}\right|,\left|V^{2}\right|\right)=0$ since $a_{1}$ and $a_{2}$ are orthogonal basis vectors. Throughout the weak-coupling regime $\left|g^{(2)}\right|<\left|g_{\mathrm{EP} 2}^{(2)}\right|, 0 \leqslant\left(\left|V^{1}\right|,\left|V^{2}\right|\right)<1$. On the other hand, in the strong-coupling regime, $\left|g^{(2)}\right|>\left|g_{\mathrm{EP} 2}^{(2)}\right|,\left(\left|V^{1}\right|,\left|V^{2}\right|\right)=1$, implying that $\left|V^{1}\right|$ and $\left|V^{2}\right|$ are parallel. While the above 
example shows how the distinct nature of eigenvectors, without any knowledge of the eigenvalues, can provide a sufficient means for distinguishing the different regimes of coupling, one should be wary of naively extending the two-mode intuition to a multimode system. For instance, one potential pitfall is to assume identical participation ratios posthybridization into supermodes as a criterion for mode indistinguishability in the strong-coupling regime. While for a two-mode system, the participation ratios indeed become identical at EP2, i.e., $\left|V_{a_{1}}^{1,2}\right|_{g=g_{\mathrm{EP} 2}^{(2)}}=\left|V_{a_{2}}^{1,2}\right|_{g=g_{\mathrm{EP} 2}^{(2)}}=1 / \sqrt{2}$, for $N>2$ systems $\left|V_{a_{j}}^{\alpha}\right| \neq 1 / \sqrt{N}$ at (or beyond) EP $N$ in general. For instance, at EP3 for $N=3$ [Fig. 2(b)], for each of the three eigenvectors $\left|V^{1,2,3}\right|=[0.42,0.71,0.57]$. In other words, $N$-way strong coupling does not guarantee equal participation of the modes in a generic $N$-mode system. We now introduce the full procedure based exclusively on eigenvectors, which reliably diagnoses strong coupling in a general $N$-mode system with bilinear interactions. Note that the modes under consideration may or may not share direct physical coupling, so the proposed method is impartial toward inclusion of interactions between non-nearest neighbor sites in the system.

(1) Consider the multiset of left eigenvectors of the mode matrix, $\mathcal{S}=\left\{V^{\alpha} \mid V^{\alpha} \mathbb{M}^{(N)}=V^{\alpha} \lambda^{\alpha}\right\}$.

(2) Define the 2-norm of each eigenvector $V^{\alpha}$, projected onto a $2 \mathrm{D}$ subspace spanned by $\left\{a_{j}, a_{k}\right\}$, as

$$
\mathcal{L}_{(j, k)}^{\alpha}=\left(\left|\left(V^{\alpha}, a_{j}\right)\right|^{2}+\left|\left(V^{\alpha}, a_{k}\right)\right|^{2}\right)^{1 / 2} .
$$

(3) Partition $\mathcal{S}$ into $m$-equivalence classes $\left[V^{\alpha}\right]_{m}$, each consisting of a set of eigenvectors with equal 2-norms for all ${ }^{N} \mathbf{C}_{2}$ projections, i.e.,

$$
\left[V^{\alpha}\right]_{m}=\left\{V^{\beta} \in \mathcal{S} \mid V^{\beta} \sim V^{\alpha}\right\},
$$

if $\mathcal{L}_{(j, k)}^{\alpha}=\mathcal{L}_{(j, k)}^{\beta}, \forall(j, k) \in[1, N]$. The size of each equivalence class defines the coupling depth, $D_{m}=\left|\left[V^{\alpha}\right]_{m}\right| \leqslant N$, for each $D_{m}$-dimensional strongly coupled subspace of the $N$-mode system.

(4) If $\left|\left[V^{\alpha}\right]_{m}\right|=1 \forall m$, this implies that all modes are weakly coupled.

(5) Two modes $a_{j}$ and $a_{k}$ are strongly coupled, if and only if

$$
\mathcal{L}_{(j, k)}^{\alpha}>\mathcal{L}_{(j, k)}^{\alpha^{\prime}} \forall V^{\alpha^{\prime}} \notin\left[V^{\alpha}\right]_{m}
$$

Using the above inequality, construct a set $\mathcal{E}_{m}$,

$$
\mathcal{E}_{m}=\left\{(j, k) ; j<k \mid \mathcal{L}_{(j, k)}^{\alpha}>\mathcal{L}_{(j, k)}^{\alpha^{\prime}}, \forall V^{\alpha^{\prime}} \notin\left[V^{\alpha}\right]_{m}\right\},
$$

whose size defines the connectivity of the subsystem, $E_{m}=$ $\left|\mathcal{E}_{m}\right|$. Connectivity represents the number of pairs of physical modes $(j, k)$ that are hybridized; i.e., each pair of modes in $\mathcal{E}_{m}$ indexes a $2 \mathrm{D}$ subspace in the $N$-dimensional (physical) mode space.

(6) The connectivity $E_{m}$ is distinct from the depth $D_{m}$ and, in general, $E_{m} \geqslant D_{m}-1$. If all pairs in $\mathcal{E}_{m}$ form a fully connected closed set, then $E_{m}={ }^{D_{m}} \mathbf{C}_{2}$ and the subspace supports an $\mathrm{EP} D_{m}$.

Note that this implies that for $D_{m}=N$, Eqs. (10) and (11) recover the condition of an EPN , i.e., coalescence of all eigenvectors of the system signifying the manifestation of $\mathrm{N}$-way strong coupling in an $\mathrm{N}$-mode system.

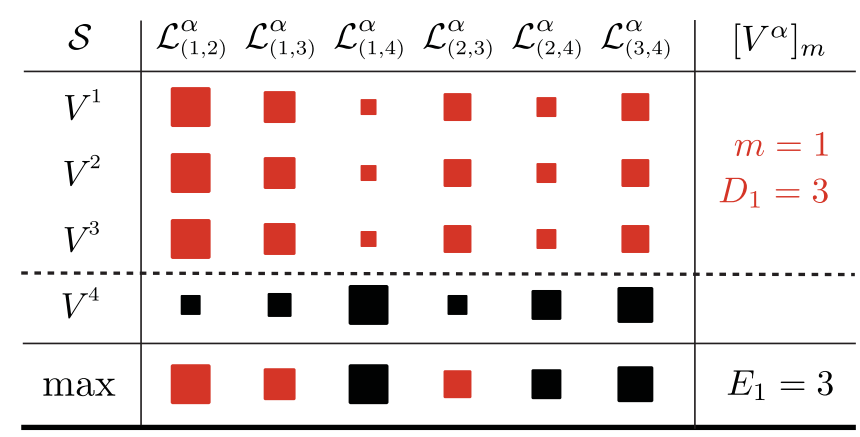

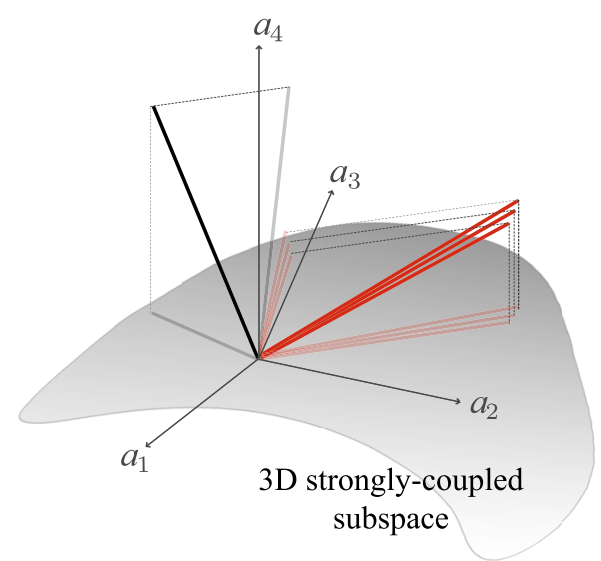

FIG. 3. (Top panel) Configuration of eigenvectors corresponding to a realization of EP3 in $N=4$ system. The size of the squares denote the magnitude of planar projections with red denoting those for strongly coupled modes and black denoting those for the weakly coupled mode. (Bottom panel) Geometric visualization of EP3 in $N=4$ system. Solid rays represent the eigenvectors while faint rays represent their corresponding projections. For clarity of presentation, only the projections in $\left\{a_{1}, a_{2}\right\}$ and $\left\{a_{3}, a_{4}\right\}$ planes are shown. The resultant EP3 creates a strongly coupled 3D subspace spanned by $\left\{a_{1}, a_{2}, a_{3}\right\}$, represented as a gray hyperplane.

The criterion prescribed in Eq. (11), which is the key result of this paper, lends itself to a helpful geometric visualization depicted in Fig. 3: Strongly coupled subspaces manifest as hyperplanes, making small angles with the equivalent eigenvectors and thus making the corresponding projections larger, while weakly coupled subspaces make large angles, leading to small projections.

We now apply this procedure to the three-mode and four-mode systems examined in Sec. II. Figure 4(a) depicts the regions where Eq. (11) holds true for each pair of modes in $N=3$ open system. For instance, in region I (red), $D=2, E=1$ with two identical eigenvectors such that $\mathcal{L}_{(1,2)}^{1}=\mathcal{L}_{(1,2)}^{2}>\mathcal{L}_{(1,2)}^{3}$ while $\mathcal{L}_{(2,3)}^{1}=\mathcal{L}_{(2,3)}^{2}<\mathcal{L}_{(2,3)}^{3}$ and $\mathcal{L}_{(1,3)}^{1}=\mathcal{L}_{(1,3)}^{2}<\mathcal{L}_{(1,3)}^{3}$, identifying this region as regime of pairwise strong coupling for modes $\left\{a_{1}, a_{2}\right\}$. Similarly, in region II (blue), $D=2, E=1$ with $\mathcal{L}_{(1,2)}^{1}=\mathcal{L}_{(1,2)}^{2}<\mathcal{L}_{(1,2)}^{3}$, $\mathcal{L}_{(2,3)}^{1}=\mathcal{L}_{(2,3)}^{2}>\mathcal{L}_{(2,3)}^{3}, \quad \mathcal{L}_{(1,3)}^{1}=\mathcal{L}_{(1,3)}^{2}<\mathcal{L}_{(1,3)}^{3}$, identifying pairwise strong coupling between modes $\left\{a_{2}, a_{3}\right\}$ in this region. Furthermore, boundaries of regions I and II delineate weak and strong coupling regimes identified using 

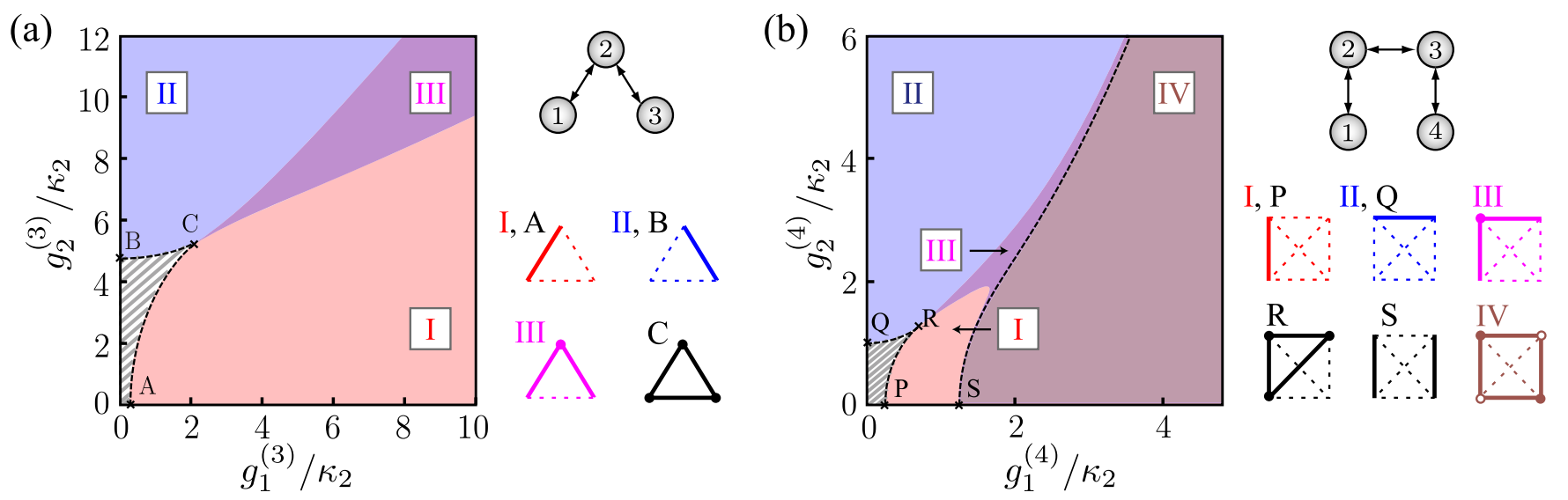

FIG. 4. Pairwise strong coupling regions calculated using Eq. (11) for (a) $N=3$ and (b) $N=4$ modes, depicted as function of respective coupling strengths. The decay rates used in each case were the same as those reported in Fig. 2. The hatched region in each plot depicts the weak-coupling region where the inequality is not satisfied for any pair of modes. The boundaries of the regions predicted by Eq. (11) coincide with the EP2 curves obtained from eigenvalues, which are reproduced here in dashed black for easy reference (cf. Fig. 2). Along with each coupling map, corresponding edge graphs show mode connectivity in each region and at EPs inferred from eigenvector-based projection, with connections between strongly coupled (weakly coupled) modes represented with solid (dashed) edges. Connected solid edges, resulting from the same equivalence class, are shown with filled circles. Disconnected solid edges denote the presence of distinct equivalence classes of dressed states, shown with empty circles for adjacent edges, for example, point $\mathrm{S}$ and region IV in panel (b).

eigenvector-based analysis, which on comparison with Fig. 2(b) are in quantitative agreement with the EP2 curves obtained from eigenvalue-based analysis. More interestingly, the proposed method identifies a region III (purple) where regions I and II overlap, i.e., $D=2, E=2$, implying simultaneous pairwise strong coupling for two pairs of modes, $\left\{a_{1}, a_{2}\right\}$ and $\left\{a_{2}, a_{3}\right\}$. Note that this does not imply that all three modes are strongly coupled in region III, because $\left\{a_{1}, a_{3}\right\}$ remain weakly coupled since $\mathcal{L}_{(1,3)}^{1}=\mathcal{L}_{(1,3)}^{2}<\mathcal{L}_{(1,3)}^{3}$ remains true in all the colored regions. In fact, the only point in parameter space $\left(g_{1}^{(3)}, g_{2}^{(3)}\right)$ that supports three-way strong coupling is point $\mathrm{C}$; here $\mathcal{L}_{(j, k)}^{1}=\mathcal{L}_{(j, k)}^{2}=\mathcal{L}_{(j, k)}^{3}$ where $(j, k) \in[1,3]$. It is worth noting that this exactly corresponds to the EP3 shown in Fig. 2(b).

The coupling phase diagram shown for $N=4$ in Fig. 4(b) is expectedly more involved. In total, there are six pairs for which we check Eq. (11), and find in

$$
\begin{aligned}
& \text { region I: } D=2, E=1 \text {, since } \\
& \qquad \mathcal{L}_{(1,2)}^{1}=\mathcal{L}_{(1,2)}^{2}>\mathcal{L}_{(1,2)}^{3,4},
\end{aligned}
$$

region II: $D=2, E=1$, since

$$
\mathcal{L}_{(2,3)}^{1}=\mathcal{L}_{(2,3)}^{2}>\mathcal{L}_{(2,3)}^{3,4},
$$

region III: $D=2, E=2$, since

$$
\bigcap_{\substack{(j, k) \in \mathcal{E} \\ \mathcal{E}=\{(1,2),(2,3)\}}} \mathcal{L}_{(j, k)}^{1}=\mathcal{L}_{(j, k)}^{2}>\mathcal{L}_{(j, k)}^{3,4} .
$$

Here, for brevity, we report only the pairs of modes that satisfy Eq. (11) for strong-coupling in the respective regions. In each region, for pairs $(j, k) \notin \mathcal{E}$,

$$
\bigcap_{(j, k) \notin \mathcal{E}} \mathcal{L}_{(j, k)}^{1}=\mathcal{L}_{(j, k)}^{2}<\mathcal{L}_{(j, k)}^{3,4} .
$$

Note that in all the regions only two-way strong coupling, i.e., $D=2$, is realized. Though more than one pair of modes are strongly coupled in regions III and IV, three- or four-way strong coupling is not realized in these regions since $\left\{a_{1}, a_{3}\right\}$ and $\left\{a_{2}, a_{4}\right\}$ are diagnosed as weakly coupled, violating the condition of full connectivity necessary for realizing higher coupling depth $D$. The transition from $S$ to region IV is particularly noteworthy, even though it entails no change in the coupling depth. Both these regions support two distinct equivalence classes of eigenvectors, each consisting of a pair of identical vectors, i.e., $D_{1}=D_{2}=2$. However, while at $S$ these support two decoupled 2D subspaces with $E_{1}=E_{2}=1$ since

$$
\begin{aligned}
\mathcal{L}_{(1,2)}^{1} & =\mathcal{L}_{(1,2)}^{2}>\mathcal{L}_{(1,2)}^{3,4} \\
\text { and } \quad \mathcal{L}_{(3,4)}^{3} & =\mathcal{L}_{(3,4)}^{4}>\mathcal{L}_{(3,4)}^{1,2},
\end{aligned}
$$

in region IV, even a very weak coupling $g_{2}$ couples these $2 \mathrm{D}$ subspaces, leading to $E_{1}=E_{2}=2$, since

$$
\begin{array}{cl} 
& \bigcap_{\substack{(j, k) \in \mathcal{E}_{1} \\
\mathcal{E}_{1}=\{(1,2),(2,3)\}}} \mathcal{L}_{(j, k)}^{1}=\mathcal{L}_{(j, k)}^{2}>\mathcal{L}_{(j, k)}^{3,4} \\
\text { and } \quad & \bigcap_{\substack{(j, k) \in \mathcal{E}_{2} \\
\mathcal{E}_{2}=\{(1,4),(3,4)\}}} \mathcal{L}_{(j, k)}^{3}=\mathcal{L}_{(j, k)}^{4}>\mathcal{L}_{(j, k)}^{1,2} .
\end{array}
$$

Thus, each equivalence class of vectors contributes a pair of adjacent edges that combine to realize four-mode hybridized states, as indicated by the respective edge diagram in Fig. 4(b). This is an open-system analogue of the superexchange interaction describing electron transfer in strongly correlated systems, where two strongly correlated electronic states can hybridize through a weakly correlated state [20]. This instance shows how information about connectivity between physical 
modes of a multimode system can reveal physics beyond that provided by coupling depth.

At point R in Fig. 4(b), $D=3, E=3$ with

$$
\bigcap_{\substack{(j, k) \in \mathcal{E} \\ \mathcal{E}=\{(1,2),(2,3),(1,3)\}}} \mathcal{L}_{(j, k)}^{1}=\mathcal{L}_{(j, k)}^{2}=\mathcal{L}_{(j, k)}^{3}>\mathcal{L}_{(j, k)}^{4},
$$

while $\mathcal{L}_{(j, k)}^{1,2,3}<\mathcal{L}_{(j, k)}^{4}$ for $(j, k)=(1,4),(2,4),(3,4)$. This diagnoses three-way strong coupling in $\left\{a_{1}, a_{2}, a_{3}\right\}$ subsystem which, as in the case of $N=3$, coincides with EP3 for this system predicted by eigenvalues [cf. Fig. 2(c)]. Further, the boundaries of different regions identified using eigenvector projections correspond exactly to the EP2 curves of Fig. 2(c) with the weak-coupling regime corresponding to the region where Eq. (11) is violated for every pair of modes. Thus, in addition to correctly predicting coordinates of EPs in parameter space, eigenvectors also provide information about which modes of system hybridize at each $\mathrm{EPN}$.

We emphasize that the preceding analysis makes exclusive use of eigenvectors, without invoking eigenvalues of the mode matrix. The proposed inequality in Eq. (11) relies on 2D projections of $N$-dimensional eigenvectors, which indicates that an analysis of pairwise-coupled subspaces is sufficient to diagnose arbitrary coupling depth in open systems with bilinear interactions. Further, eigenvector-based analysis supersedes the information obtained from usual EP physics unraveled by eigenvalues, by providing means to identify physical modes defining the strongly coupled subsystems in a multimode system.

\section{APPLICATION: DISSIPATION-ENGINEERED COOLING}

In this section, we elucidate the physical implications of the eigenvector-based strong-coupling diagnostic by applying it to the problem of quantum ground-state cooling. Cooling quantum systems is a mainstay in many quantum information platforms where a mode (or qubit) needs to be prepared in its ground state (or "reset"). For instance, in conventional optomechanical platforms, a hot mechanical oscillator $\left(a_{1}\right)$ is parametrically coupled to a cold optical resonator $\left(a_{2}\right)$ that acts as an engineered reservoir. On modulating the coupling at the difference frequency of the two modes, the mechanical mode is cooled by shuttling excitations to a quickly decaying optical mode. The resultant phonon population in the steady state for the resolved sideband regime is [21]

$$
\begin{aligned}
n_{1}^{(2)} & =n_{m} \frac{1+\kappa_{1} / \kappa_{2}\left(1+\mathcal{C}_{1}\right)}{\left(1+\kappa_{1} / \kappa_{2}\right)\left(1+\mathcal{C}_{1}\right)}+n_{o} \frac{\mathcal{C}_{1}}{\left(1+\kappa_{1} / \kappa_{2}\right)\left(1+\mathcal{C}_{1}\right)} \\
& \approx n_{m}\left(\kappa_{1} / \kappa_{2}+1 / \mathcal{C}_{1}\right)+n_{o},
\end{aligned}
$$

where $\kappa_{1,2}$ denote the decay rates associated with the mechanical and optical modes, $n_{m}$ and $n_{o}$ denote their respective thermal populations in the absence of coupling, and the coupling strength $g_{1}$ is parametrized in terms of cooperativity $\mathcal{C}_{1}=4 g_{1}^{2} / \kappa_{1} \kappa_{2}$. From the simplified expression obtained in the limit of large cooperativity $\mathcal{C}_{1} \gg 1$ and the typical decay hierarchy $\kappa_{1} / \kappa_{2} \ll 1$, we can identify two distinct regimes of operation: (i) cooperativity-dominated

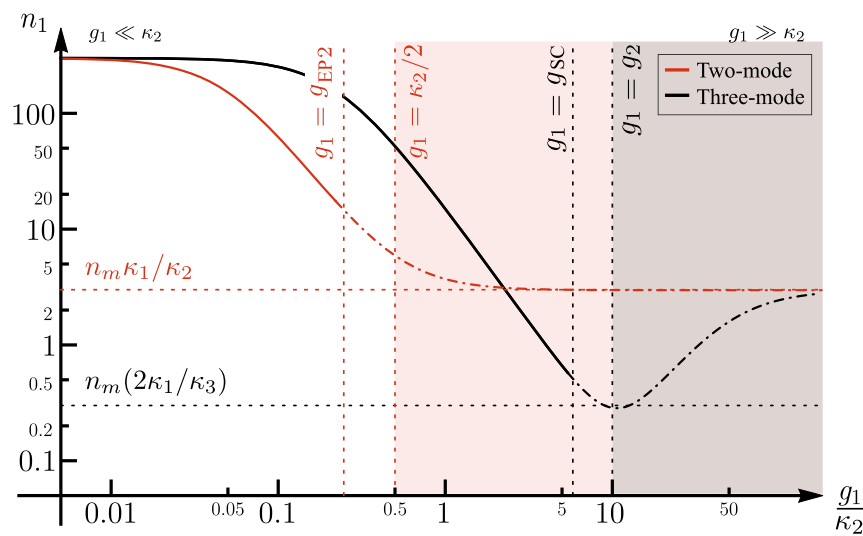

FIG. 5. Phonon population vs coupling strength for the twomode $\left(g_{2}=0\right)$ and three-mode $\left(g_{2} / \kappa_{2}=10, \mathcal{C}_{2}=20\right)$ systems with $n_{m}=300, n_{0}=n_{a}=0.1$, calculated for the same decay rates as used in Fig. 2(b). The red (gray) region represents the decaydominated regime for the two-mode (three-mode) system, calculated using expression for phonon population in Eq. (13) [(16)]. The horizontal dashed lines correspond to the minimum achievable population, while the vertical dashed lines correspond to critical $g_{1}$ values, indicating the onset of strong coupling and the decay-dominated regimes. For each system, the population is shown with dash-dotted curves in the regime where the mechanical mode is hybridized with optics. The onset of this region for the two-mode system $\left(g_{\mathrm{EP} 2}\right)$ is estimated using the eigenvalues [cf. Fig. 2(b)], while for the three-mode system $\left(g_{\mathrm{SC}}\right)$ it is estimated using the eigenvector projection-based method introduced in Sec. III [cf. Fig. 4].

regime, or $\kappa_{1} / \kappa_{2} \ll 1 / \mathcal{C}_{1}$, and (ii) decay-dominated regime, or $\kappa_{1} / \kappa_{2} \gg 1 / \mathcal{C}_{1}$. As is evident from the red curve in Fig. 5, the mechanical mode experiences active cooling as long as the system is the cooperativity-dominated regime. For coupling strengths $g_{1} / \kappa_{2}>1$, the population becomes independent of $g_{1}$ and saturates to the steady-state value determined by bare decay rates $n_{1, \min }^{(2)}=n_{m}\left(\kappa_{1} / \kappa_{2}\right)$. This crossover into dissipation-dominated regime is intimately related to the onset of strong coupling and hybridization of the mechanical and optical modes at $g_{1}=g_{\mathrm{EP} 2}$, which eventually manifests as saturation of phonon population [22].

The threshold for this crossover into strong coupling can be modified by coupling the mechanical mode to a more complex bath. The minimal system to implement this is the three-mode system considered in Sec. II, where a second optical mode $a_{3}$ is introduced as an additional auxiliary reservoir with no direct coupling to the mechanics $a_{1}$. The goal is to delimit the regime where the target system $\left(a_{1}\right)$ remains weakly coupled with the system of engineered reservoir modes $\left(a_{2}, a_{3}\right)$, in order to extend the cooperativity-dominated regime for cooling. Based on the coupling phase diagram of Fig. 4(a), this may be achieved if we choose to operate in region II where $\left\{a_{1}, a_{2}\right\}$ and $\left\{a_{1}, a_{3}\right\}$ subsystems remain weakly coupled, while optical baths $a_{1}$ and $a_{2}$ hybridize to form supermodes.

To demonstrate this, we follow the same procedure as for the two-mode case and calculate the phonon population as a function of coupling of the mechanics to the system of optical cavities $g_{1}$. To gain some intuition of the modified strong coupling threshold, we first treat the auxiliary optical mode 
$a_{3}$ as quasistatic, $\kappa_{3} \gg \max \left\{\kappa_{1}, \kappa_{2}\right\}$, and use its steady-state solution,

$$
a_{3}=\frac{2}{\kappa_{3}}\left(-i g_{2} a_{2}+\sqrt{\kappa_{3}} a_{3}^{\text {in }}\right),
$$

to solve for dynamics of the reduced two-mode system $\left\{a_{1}, a_{2}\right\}$. In this limit, the mechanical mode can be viewed as being coupled to a single optical mode $a_{2}$ with a modified decay rate $\kappa_{2}^{\text {eff }}=\kappa_{2}\left(1+\mathcal{C}_{2}\right)$, and a concomitant input noise $a_{2}^{\text {in,eff }}=a_{2}^{\text {in }}-i \sqrt{\mathcal{C}_{2}} a_{3}^{\text {in }}$, where $\mathcal{C}_{2}=4 g_{2}^{2} /\left(\kappa_{2} \kappa_{3}\right)$ denotes the cooperativity for the optical subsystem. Following standard procedure, we find the phonon population for this effective two-mode system as

$$
\begin{aligned}
n_{1}^{(2), \mathrm{eff}}= & n_{m} \frac{\left(1+\mathcal{C}_{2}\right)+\kappa_{1} / \kappa_{2}^{\text {eff }}\left(1+\mathcal{C}_{1}+\mathcal{C}_{2}\right)}{\left(1+\kappa_{1} / \kappa_{2}^{\text {eff }}\right)\left(1+\mathcal{C}_{1}+\mathcal{C}_{2}\right)} \\
& +n_{o} \frac{\mathcal{C}_{1} /\left(1+\mathcal{C}_{2}\right)}{\left(1+\kappa_{1} / \kappa_{2}^{\text {eff }}\right)\left(1+\mathcal{C}_{1}+\mathcal{C}_{2}\right)} \\
& +n_{a} \frac{\mathcal{C}_{1} \mathcal{C}_{2} /\left(1+\mathcal{C}_{2}\right)}{\left(1+\kappa_{1} / \kappa_{2}^{\text {eff }}\right)\left(1+\mathcal{C}_{1}+\mathcal{C}_{2}\right)},
\end{aligned}
$$

where $n_{m}, n_{o}$, and $n_{a}$ denote the intrinsic populations of the mechanical mode and optical modes in the absence of couplings. In the limit of $\mathcal{C}_{1} \rightarrow \infty, n_{1, \text { min }}^{(2) \text { eff }}=n_{m}\left(\kappa_{1} / \kappa_{2}^{\text {eff }}\right)$, analogous to the conventional two-mode system. This simple analysis indicates that in the presence of an additional decay channel presented by the auxiliary mode $a_{3}$, strong-coupling threshold may be realized at a higher value corresponding to the high effective decay rate presented by the bath modes. However, an adiabatic elimination of $a_{3}$ strictly holds true for $\mathcal{C}_{2} \leqslant 1$. In order to obtain phonon population for strong coupling between optical modes-which is the regime of interest for operating in region II of Fig. 4(a)-we perform the calculation for the full three-mode system including the dynamics of the auxiliary optical mode. For full details of this calculation, we refer the reader to Appendix B; here we present the simplified expression for phonon population, obtained in the limit of large cooperativities $\left(\mathcal{C}_{1,2} \gg 1\right)$ and for the decay hierarchy $\kappa_{3} \gg \kappa_{2}>\kappa_{1}$,

$$
\begin{aligned}
n_{1}^{(3)} \approx & n_{m}\left(\frac{\mathcal{C}_{2}}{\mathcal{C}_{1}+\mathcal{C}_{2}}+\frac{\kappa_{1}^{2}}{\kappa_{3}^{2}} \frac{\mathcal{C}_{1}}{\mathcal{C}_{2}}\right)+n_{o}\left(\frac{\mathcal{C}_{1}}{\mathcal{C}_{1}+\left(\kappa_{3}^{2} / \kappa_{1} \kappa_{2}\right) \mathcal{C}_{2}}\right) \\
& +n_{a}\left(\frac{\left(\kappa_{3}^{2} / \kappa_{1} \kappa_{2}\right) \mathcal{C}_{2}}{\mathcal{C}_{1}+\left(\kappa_{3}^{2} / \kappa_{1} \kappa_{2}\right) \mathcal{C}_{2}}\right) .
\end{aligned}
$$

Following a similar line of logic as for the two-mode case, we can distinguish the cooperativity-dominated regime $\left(\mathcal{C}_{2} / \mathcal{C}_{1} \gg\right.$ $\left.\kappa_{1} / \kappa_{3}\right)$ from the decay-dominated regime $\left(\mathcal{C}_{2} / \mathcal{C}_{1} \ll \kappa_{1} / \kappa_{3}\right)$ of operation by analyzing the coefficient of the $n_{m}$ term. Interestingly, the crossover between these two regimes is realized when the two couplings are balanced, i.e., $g_{1}=g_{2}$. As shown by the result of the full calculation (black curve in Fig. 5), this is also the point where the lowest phonon population is achieved with the floor, $n_{1, \min }^{(3)}=2 n_{m} \kappa_{1} / \kappa_{3}$, determined solely by the decay rates. This indicates that while the quantum correlations of reservoir modes enhance cooling, eventually strong-coupling effects lead to a resurgence observed for large values of $g_{1}$.
Note that unlike the two-mode case where the real and imaginary parts of eigenvalues exhibit a bifurcation as the system crosses EP2, the eigenvalues of $\mathbb{M}^{(3)}$ show no characteristic signature as this crossover is approached [see Fig. 2(b)]. However, we can evaluate a threshold value for $g_{1}$, given a value of $g_{2}$, using the metric proposed in Eq. (11), below which mechanical mode remains weakly coupled. This value of $g_{1}=g_{\mathrm{SC}}$ corresponds to the intersection of the line $g_{2} / \kappa_{2}=10$ with the boundary of regions II and III in Fig. 4(a). Notably, as shown in Fig. 5, the predicted value of $g_{S C}$ is consistent with the fact that hybridization of the modes acts as a precursor for population saturation, and beyond this point the cooling is progressively impeded with increase in coupling. Thus, eigenvector-based analysis is able to detect the transition from weak to strong coupling in dissipation-engineered systems, which cannot be discerned by analyzing eigenvalues.

\section{CONCLUSIONS}

In conclusion, we have introduced a method to diagnose strong coupling in a multimode open system with bilinear interactions. The proposed method is based entirely on eigenvectors of the matrix describing the coupling and local decay rates of the modes. In addition to delineating the regions of weak and strong coupling, it allows a means to identify the physical subsystems that undergo hybridization in different regions of the coupling landscape and shows how different connectivity configurations can be present while maintaining a fixed coupling depth. This indicates that detailed information about both connectivity and coupling depth is essential for a full characterization of dressed states in strongly coupled systems. We present sideband cooling in a multimode optomechanical system as an example to show how this method can reveal the crossover of the target oscillator from cooperativitydominated dynamics to decay-dominated dynamics in the presence of a strongly hybridized optical reservoir. Thus, using eigenvectors to characterize open system dynamics, which cannot be detected by EPs, can present opportunities for dissipation engineering where, by construction or design, only a subsystem is accessible for control and measurement.

Remarkably, the proposed method shows how tiling only pairwise hybridized modes can detect exceptional points of arbitrary order (at least for bilinear interactions). This is strikingly reminiscent of dimensional reduction methods used for feature analysis of multidimensional data and bodes well for the ongoing program of using low-weight measurements to deduce the structure of complex entangled states. The current work thus just scratches the surface in adapting sophisticated data analysis tools to resolve challenging problems in many-body open systems. For instance, leveraging connections to statistical techniques such as projection pursuit, the eigenvector-based method presented here may be generalized to different coupling topologies, $P T$-symmetric systems [23,24], systems with gain [25], and even nonlinear couplings. Finally, our results present an interesting counterpoint to recent proofs of eigenvector-eigenvalue identity proven for Hermitian matrices [26] and suggest that information parity between eigenvalues and eigenvectors may not hold for opensystem physics described by complex symmetric matrices, even in principle. 


\section{ACKNOWLEDGMENTS}

The authors wish to thank John Teufel, Hakan E. Türeci, Jamie Kerman, and Emery Doucet for useful conversations. We also thank Tristan Brown and K. S. Sreekara Sabarish for their careful reading of the manuscript. This research was supported by the U.S. Department of Energy under Grants No. DE-SC0019515 (C.K.) and No. DE-SC0019461 (Z.X.). A.M. acknowledges funding by the Deutsche Forschungsgemeinschaft through the Emmy Noether program (Grant No. ME 4863/1-1) and the project CRC 910.

\section{APPENDIX A: EIGENVALUE ANALYSIS FOR THREE- AND FOUR-MODE SYSTEMS}

\section{1. $N=3$ case}

We first write the characteristic polynomial $p(\lambda)$ of $\mathbb{M}^{(3)}$, as $p(\lambda)=\alpha \lambda^{3}+\beta \lambda^{2}+\gamma \lambda+\delta$, where

$$
\begin{aligned}
& \alpha=1, \\
& \beta=\frac{\kappa_{1}+\kappa_{2}+\kappa_{3}}{2}, \\
& \gamma=g_{1}^{(3) 2}+g_{2}^{(3) 2}+\frac{\kappa_{1} \kappa_{2}+\kappa_{1} \kappa_{3}+\kappa_{2} \kappa_{3}}{4}, \\
& \delta=\frac{4 g_{1}^{(3) 2} \kappa_{3}+4 g_{2}^{(3) 2} \kappa_{1}+\kappa_{1} \kappa_{2} \kappa_{3}}{8} .
\end{aligned}
$$

Using Cardano's method, we can first write $p(x)$ into the depressed cubic form $p^{\prime}(t)$ by substituting $\lambda=t-\beta / 3 \alpha$, such that

$$
p^{\prime}(t)=t^{3}+3 \epsilon_{1} t-2 \epsilon_{2},
$$

with

$$
\begin{aligned}
\epsilon_{1} & =\frac{3 \alpha \gamma-\beta^{2}}{9 \alpha^{2}}, \\
\epsilon_{2} & =\frac{9 \alpha \beta \gamma-27 \alpha^{2} \delta-2 \beta^{3}}{54 \alpha^{3}} .
\end{aligned}
$$

Solving for the roots of the cubic equation, $p^{\prime}(t)=0$, and using Eqs. (A3) give the eigenvalues $e_{i}^{(3)}$ of $\mathbb{M}^{(3)}$ as

$$
\begin{aligned}
& e_{1}^{(3)}=\eta_{0}+\eta_{+}+\eta_{-}, \\
& e_{2}^{(3)}=\eta_{0}+e^{i 2 \pi / 3} \eta_{+}+e^{i 4 \pi / 3} \eta_{-}, \\
& e_{3}^{(3)}=\eta_{0}+e^{i 4 \pi / 3} \eta_{+}+e^{i 2 \pi / 3} \eta_{-},
\end{aligned}
$$

where $\eta_{0}=-\beta /(3 \alpha), \eta_{ \pm}=\left(\epsilon_{2} \pm{\sqrt{\epsilon_{2}^{2}+\epsilon_{1}^{3}}}^{1 / 3}\right.$. In this representation, the location of exceptional points can be found as [27]

$$
\begin{gathered}
\operatorname{EP} 2: \operatorname{disc}\left(\mathbb{M}^{(3)}\right)=0 \Longleftrightarrow \epsilon_{1}^{2}+\epsilon_{2}^{3}=0 \\
\operatorname{EP} 3: \epsilon_{1}=0 \text { and } \epsilon_{2}=0 .
\end{gathered}
$$

\section{2. $N=4$ case}

For the four-mode case, we similarly write the characteristic polynomial of $\mathbb{M}^{(4)}$ as $p(\lambda)=a \lambda^{4}+b \lambda^{3}+c \lambda^{2}+d \lambda+e$, where

$$
\begin{aligned}
a= & 1 \\
b= & \frac{\kappa_{1}+\kappa_{2}+\kappa_{3}+\kappa_{4}}{2}, \\
c= & 2 g_{1}^{(4) 2}+g_{2}^{(4) 2} \\
& +\frac{\kappa_{1} \kappa_{2}+\kappa_{1} \kappa_{3}+\kappa_{2} \kappa_{3}+\kappa_{1} \kappa_{4}+\kappa_{2} \kappa_{4}+\kappa_{3} \kappa_{4}}{4} \\
d= & \frac{1}{8}\left(4 g_{1}^{(4) 2}\left(\kappa_{1}+\kappa_{2}+\kappa_{3}+\kappa_{4}\right)+4 g_{2}^{(4) 2}\left(\kappa_{1}+\kappa_{4}\right)\right. \\
& \left.+\kappa_{1} \kappa_{2} \kappa_{3}+\kappa_{1} \kappa_{2} \kappa_{4}+\kappa_{1} \kappa_{3} \kappa_{4}+\kappa_{2} \kappa_{3} \kappa_{4}\right) \\
e= & g_{1}^{(4) 4}+\frac{1}{4} g_{1}^{(4) 2}\left(\kappa_{1} \kappa_{2}+\kappa_{3} \kappa_{4}\right) \\
& +\frac{1}{16} \kappa_{1} \kappa_{4}\left(4 g_{2}^{(4) 2}+\kappa_{2} \kappa_{3}\right) .
\end{aligned}
$$

Using Ferrari's method, we rewrite $p(\lambda)$ in depressed quartic form $P^{\prime}$ by substituting $\lambda=y-b /(4 a)$ such that

$$
P^{\prime}(y)=y^{4}+f_{1} y^{2}+f_{2} y+f_{3},
$$

where

$$
\begin{aligned}
& f_{1}=\frac{8 a c-3 b^{2}}{8 a^{2}}, \\
& f_{2}=\frac{b^{3}-4 a b c+8 a^{2} d}{8 a^{3}}, \\
& f_{3}=\frac{-3 b^{4}+16 a b^{2} c-64 a^{2} b d+256 a^{3} e}{256 a^{4}} .
\end{aligned}
$$

Solving for $y$ and subsequently $\lambda$ gives the eigenvalues of $\mathbb{M}^{(4)}$ as

$$
\begin{aligned}
& e_{1}^{(4)}=G_{1}-G_{3}+\sqrt{-G_{3}^{2}-\frac{f_{1}}{2}+\frac{f_{2}}{4 G_{3}}}, \\
& e_{2}^{(4)}=G_{1}-G_{3}-\sqrt{-G_{3}^{2}-\frac{f_{1}}{2}+\frac{f_{2}}{4 G_{3}}}, \\
& e_{3}^{(4)}=G_{1}+G_{3}+\sqrt{-G_{3}^{2}-\frac{f_{1}}{2}-\frac{f_{2}}{4 G_{3}}}, \\
& e_{4}^{(4)}=G_{1}+G_{3}-\sqrt{-G_{3}^{2}-\frac{f_{1}}{2}-\frac{f_{2}}{4 G_{3}}},
\end{aligned}
$$

where

$$
\begin{aligned}
G_{1} & =-\frac{b}{4 a}, \\
G_{2} & =\left(\frac{g_{1}+\sqrt{g_{1}^{2}-4 g_{2}^{3}}}{2}\right)^{1 / 3}, \\
G_{3} & =\frac{1}{2} \sqrt{g_{3}+\frac{1}{3}\left(G_{2}+\frac{g_{2}}{G_{2}}\right)}
\end{aligned}
$$




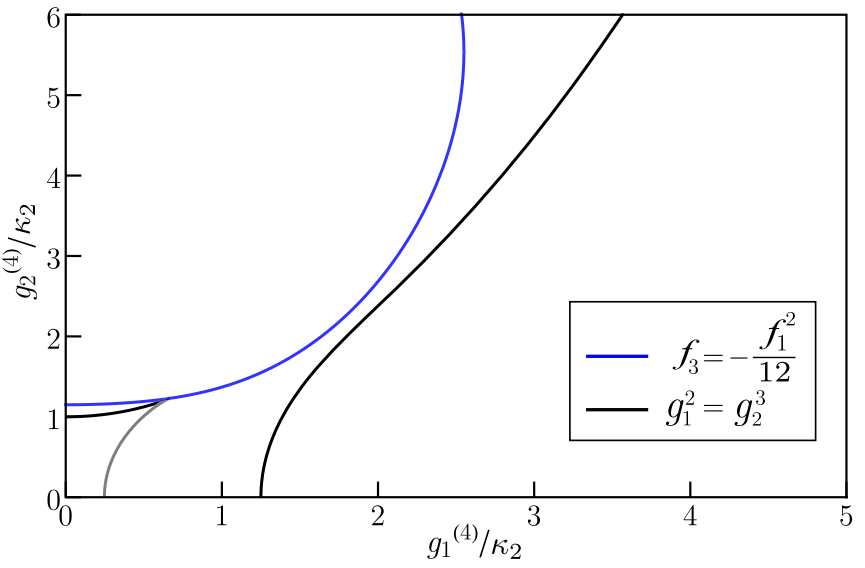

FIG. 6. Black curves denote locus of EP2 points obtained using $\operatorname{disc}\left(\mathbb{M}^{(4)}\right)=0$, while the blue curve is a parametric plot of the second condition in Eq. (A14) calculated for the decay rates used in Fig. 2(c). The intersection of the black and blue curves gives the location of EP3 for the four-mode system.

with

$$
\begin{aligned}
& g_{1}=\frac{2 c^{3}-9 b c d+27 b^{2} e+27 a d^{2}-72 a c e}{a^{3}}, \\
& g_{2}=\frac{c^{2}-3 b d+12 a e}{a^{2}}, \\
& g_{3}=\frac{3 b^{2}-8 a c}{12 a^{2}} .
\end{aligned}
$$

In this representation, the location for exceptional points follows similarly as in the $N=3$ case:

$$
\mathrm{EP} 2: \operatorname{disc}\left(\mathbb{M}^{(4)}\right)=0 \Longleftrightarrow g_{1}^{2}-4 g_{2}^{3}=0 .
$$

However, for EP3 in the four-mode system,

$$
g_{1}^{2}=4 g_{2}^{3} \text { and } f_{3}=-f_{1}^{2} / 12 .
$$

We note that for the decay rates used in the main text, exactly one EP3 is realized for the $N=4$ system as depicted in Fig. 6.

\section{APPENDIX B: CALCULATIONS FOR THREE-MODE COOLING}

Using the Hamiltonian in Eq. (1) for $N=3$, we can write the equations of motion for a three-mode optomechanical system as

$$
\begin{aligned}
& \frac{d \hat{a}_{1}}{d t}=-i g_{1} \hat{a}_{2}-\frac{\kappa_{1}}{2} \hat{a}_{1}+\sqrt{\kappa_{1}} \hat{a}_{1}^{\text {in }}, \\
& \frac{d \hat{a}_{2}}{d t}=-i g_{1} \hat{a}_{1}-i g_{2} \hat{a}_{3}-\frac{\kappa_{2}}{2} \hat{a}_{2}+\sqrt{\kappa_{2}} \hat{a}_{2}^{\text {in }},
\end{aligned}
$$

$$
\frac{d \hat{a}_{3}}{d t}=-i g_{2} \hat{a}_{2}-\frac{\kappa_{3}}{2} \hat{a}_{3}+\sqrt{\kappa_{3}} \hat{a}_{3}^{\text {in }} .
$$

This system of coupled differential equations can be solved as a system of algebraic equations in Fourier domain to obtain the solution for the mechanical mode operator $a_{1}[\omega]$,

$$
\begin{aligned}
\hat{a}_{1}[\omega]= & \sqrt{\kappa_{1}}\left\{\frac{\left(g_{2}^{2}+\chi_{2}^{-1} \chi_{3}^{-1}\right)}{g_{1}^{2} \chi_{3}^{-1}+\chi_{1}^{-1}\left(g_{2}^{2}+\chi_{2}^{-1} \chi_{3}^{-1}\right)}\right\} \hat{a}_{1}^{\text {in }}[\omega] \\
& +\sqrt{\kappa_{2}}\left\{\frac{-i g_{1} \chi_{3}^{-1}}{g_{1}^{2} \chi_{3}^{-1}+\chi_{1}^{-1}\left(g_{2}^{2}+\chi_{2}^{-1} \chi_{3}^{-1}\right)}\right\} \hat{a}_{2}^{\text {in }}[\omega] \\
& +\sqrt{\kappa_{3}}\left\{\frac{-g_{1} g_{2}}{g_{1}^{2} \chi_{3}^{-1}+\chi_{1}^{-1}\left(g_{2}^{2}+\chi_{2}^{-1} \chi_{3}^{-1}\right)}\right\} \hat{a}_{3}^{\text {in }}[\omega],
\end{aligned}
$$

with the susceptibilities $\chi_{i}^{-1}=-i \omega+\kappa_{i} / 2, i \in\{1,2,3\}$. This enables calculation of the symmetrized spectral density for the mechanical mode,

$$
\bar{S}_{\hat{a}_{1} \hat{a}_{1}}^{(3)}[\omega]=\frac{1}{2} \int_{-\infty}^{\infty} d \omega^{\prime}\left\langle\hat{a}_{1}^{\dagger}[\omega] \hat{a}_{1}\left[\omega^{\prime}\right]+\hat{a}_{1}[\omega] \hat{a}_{1}^{\dagger}\left[\omega^{\prime}\right]\right\rangle,
$$

from which effective population of the mechanical mode then follows as

$$
\left(n_{1}^{(3)}+\frac{1}{2}\right)=\int \frac{d \omega}{2 \pi} \bar{S}_{\hat{a}_{1} \hat{a}_{1}}[\omega] .
$$

The resultant expression of $\bar{n}_{1}^{(3)}$ obtained following this procedure is

$$
n_{1}^{(3)}=n_{m} \frac{\sigma_{1}}{\Sigma}+n_{o} \frac{\sigma_{2}}{\Sigma}+n_{a} \frac{\sigma_{3}}{\Sigma},
$$

where

$$
\begin{aligned}
\sigma_{1}= & {\left[\frac{\kappa_{1}^{2}}{\kappa_{\|}^{2}}+\frac{\kappa_{1}^{3}}{\kappa_{2} \kappa_{\|} \kappa_{\perp}}+\frac{\kappa_{1}^{3}}{\kappa_{3} \kappa_{\|} \kappa_{\perp}}\left(1+\mathcal{C}_{1}\right)\right]\left(1+\mathcal{C}_{1}+\mathcal{C}_{2}\right) } \\
& +\left(\frac{\kappa_{1}}{\kappa_{\|}}\right)\left(1+\mathcal{C}_{2}\right)^{2}-\left(\frac{\kappa_{1}^{2}}{\kappa_{\|} \kappa_{\perp}}\right) \mathcal{C}_{1}\left(1+\mathcal{C}_{2}\right), \\
\sigma_{2}= & \left(\frac{\kappa_{1}}{\kappa_{\|}}\right) \mathcal{C}_{1}+\left(\frac{\kappa_{1}^{2}}{\kappa_{3}^{2}}\right) \mathcal{C}_{1}\left(\mathcal{C}_{1}+\mathcal{C}_{2}+\frac{\kappa_{3}}{\kappa_{\|}}\right), \\
\sigma_{3}= & \left(\frac{\kappa_{1}}{\kappa_{\|}}+\frac{\kappa_{1}^{2}}{\kappa_{\|} \kappa_{\perp}}\right) \mathcal{C}_{1} \mathcal{C}_{2}, \\
\Sigma= & \left(1+\mathcal{C}_{1}+\mathcal{C}_{2}\right)\left[\frac{\kappa_{1}}{\kappa_{\|}}\left(1+\mathcal{C}_{2}\right)+\frac{\kappa_{1}^{2}}{\kappa_{2}^{3}} \mathcal{C}_{1}\left(1+\frac{\kappa_{1} \kappa_{3}}{\kappa_{\|} \kappa_{\perp}}\right)\right. \\
& \left.+\frac{\kappa_{1}^{2}}{\kappa_{\|}^{2} \kappa_{\perp}}\left(\kappa_{1}+\kappa_{\perp}\right)\right],
\end{aligned}
$$

with $\kappa_{\|}=\kappa_{2} \kappa_{3} /\left(\kappa_{2}+\kappa_{3}\right), \kappa_{\perp}=\kappa_{2}+\kappa_{3}$.
[1] B. Peng, Ş. K. Özdemir, M. Liertzer, W. Chen, J. Kramer, H. Yilmaz, J. Wiersig, S. Rotter, and L. Yang, Chiral modes and directional lasing at exceptional points, Proc. Natl. Acad. Sci. USA 113, 6845 (2016).

[2] J. W. Yoon, Y. Choi, C. Hahn, G. Kim, S. H. Song, K.-Y. Yang, J. Y. Lee, Y. Kim, C. S. Lee, J. K. Shin, H.-S. Lee, and P. Berini,
Time-asymmetric loop around an exceptional point over the full optical communications band, Nature (London) 562, 86 (2018).

[3] S. A. Hassani Gangaraj and F. Monticone, Topological Waveguiding Near an Exceptional Point: Defect-Immune, Slow-Light, and Loss-Immune Propagation, Phys. Rev. Lett. 121, 093901 (2018). 
[4] Y. Choi, C. Hahn, J. W. Yoon, S. H. Song, and P. Berini, Extremely broadband, on-chip optical nonreciprocity enabled by mimicking nonlinear anti-adiabatic quantum jumps near exceptional points, Nat. Commun. 8, 14154 (2017).

[5] Q. Zhong, S. Ozdemir, A. Eisfeld, A. Metelmann, and R. ElGanainy, Exceptional-Point-Based Optical Amplifiers, Phys. Rev. Appl. 13, 014070 (2020).

[6] J. Wiersig, Enhancing the Sensitivity of Frequency and Energy Splitting Detection by Using Exceptional Points: Application to Microcavity Sensors for Single-Particle Detection, Phys. Rev. Lett. 112, 203901 (2014).

[7] W. Chen, A. K. Özdemir, G. Zhao, J. Wiersig, and L. Yang, Exceptional points enhance sensing in an optical microcavity, Nature (London) 548, 192 (2017).

[8] M. Zhang, W. Sweeney, C. W. Hsu, L. Yang, A. D. Stone, and L. Jiang, Quantum Noise Theory of Exceptional Point Amplifying Sensors, Phys. Rev. Lett. 123, 180501 (2019).

[9] L. Feng, Z. J. Wong, R.-M. Ma, Y. Wang, and X. Zhang, Singlemode laser by parity-time symmetry breaking, Science 346, 972 (2014).

[10] H. Hodaei, M.-A. Miri, M. Heinrich, D. N. Christodoulides, and M. Khajavikhan, Parity-time-symmetric microring lasers, Science 346, 975 (2014).

[11] B. Peng, Ş. K. Özdemir, S. Rotter, H. Yilmaz, M. Liertzer, F. Monifi, C. M. Bender, F. Nori, and L. Yang, Lossinduced suppression and revival of lasing, Science 346, 328 (2014).

[12] A. P. Seyranian, O. N. Kirillov, and A. A. Mailybaev, Coupling of eigenvalues of complex matrices at diabolic and exceptional points, J. Phys. A: Math. Gen. 38, 1723 (2005).

[13] T. J. Milburn, J. Doppler, C. A. Holmes, S. Portolan, S. Rotter, and P. Rabl, General description of quasiadiabatic dynamical phenomena near exceptional points, Phys. Rev. A 92, 052124 (2015).
[14] H. Xu, D. Mason, L. Jiang, and J. G. E. Harris, Topological energy transfer in an optomechanical system with exceptional points, Nature (London) 537, 80 (2016).

[15] W. D. Heiss, Phases of wave functions and level repulsion, Eur. Phys. J. D 7, 1 (1999).

[16] Technically, $p_{\mathbb{M}^{(N)}}$ needs to be the minimal polynomial of the mode matrix.

[17] W. P. Su, J. R. Schrieffer, and A. J. Heeger, Solitons in Polyacetylene, Phys. Rev. Lett. 42, 1698 (1979).

[18] A. J. Heeger, S. Kivelson, J. R. Schrieffer, and W. P. Su, Solitons in conducting polymers, Rev. Mod. Phys. 60, 781 (1988).

[19] L. Li, Z. Xu, and S. Chen, Topological phases of generalized Su-Schrieffer-Heeger models, Phys. Rev. B 89, 085111 (2014).

[20] J. Kanamori, Superexchange interaction and symmetry properties of electron orbitals, J. Phys. Chem. Solids 10, 87 (1959).

[21] M. Aspelmeyer, T. J. Kippenberg, and F. Marquardt, Cavity optomechanics, Rev. Mod. Phys. 86, 1391 (2014).

[22] J. M. Dobrindt, I. Wilson-Rae, and T. J. Kippenberg, Parametric Normal-Mode Splitting in Cavity Optomechanics, Phys. Rev. Lett. 101, 263602 (2008).

[23] C. M. Bender and S. Boettcher, Real Spectra in Non-Hermitian Hamiltonians Having $\mathscr{P} \mathscr{T}$ Symmetry, Phys. Rev. Lett. 80, 5243 (1998).

[24] R. El-Ganainy, K. G. Makris, M. Khajavikhan, Z. H. Musslimani, S. Rotter, and D. N. Christodoulides, NonHermitian physics and PT symmetry, Nat. Phys. 14, 11 (2018).

[25] M.-A. Miri and A. Alù, Exceptional points in optics and photonics, Science 363, eaar7709 (2019).

[26] P. B. Denton, S. J. Parke, T. Tao, and X. Zhang, Eigenvectors from eigenvalues: A survey of a basic identity in linear algebra, Bull. Amer. Math. Soc. (2021), doi:10.1090/bull/1722.

[27] M. Am-Shallem, R. Kosloff, and N. Moiseyev, Exceptional points for parameter estimation in open quantum systems: Analysis of the Bloch equations, New J. Phys. 17, 113036 (2015). 